\title{
Magnetic neutrino scattering on atomic electrons revisited
}

\author{
Konstantin A. Kouzakov ${ }^{\mathrm{a}, *}$, Alexander I. Studenikin ${ }^{\mathrm{b}, \mathrm{c}}$ \\ ${ }^{a}$ Department of Nuclear Physics and Quantum Theory of Collisions, Faculty of Physics, \\ Moscow State University, Moscow 119991, Russia \\ ${ }^{b}$ Department of Theoretical Physics, Faculty of Physics, Moscow State University, \\ Moscow 119991, Russia \\ ${ }^{c}$ Joint Institute for Nuclear Research, Dubna 141980, Moscow Region, Russia
}

\begin{abstract}
We reexamine the role of electron binding effects in the inelastic neutrinoatom scattering induced by the neutrino magnetic moment. The differential cross section of the process is presented as a sum of the longitudinal and transverse components, according to whether the force that the neutrino magnetic moment exerts on electrons is parallel or perpendicular to momentum transfer. The atomic electrons are treated nonrelativistically. On this basis, the recent theoretical predictions concerning the magnetic neutrinoimpact ionization of atoms are critically discussed. Numerical calculations are performed for ionization of a hydrogenlike $\mathrm{Ge}^{+31}$ ion by neutrino impact.
\end{abstract}

Keywords: neutrino magnetic moment, neutrino-impact ionization

\section{Introduction}

Neutrino electromagnetic properties are among the most intriguing issues in neutrino physics (see Ref. [1] for a review on this subject). Within the Standard Model the value of the neutrino magnetic moment in units of the Bohr magneton $\mu_{B}=e /\left(2 m_{e}\right)$ is $[2, \underline{3}, 4]$

$$
\mu_{\nu} \approx 3.2 \times 10^{-19}\left(\frac{m_{\nu}}{1 \mathrm{eV}}\right)
$$

\footnotetext{
${ }^{*}$ Corresponding author

Email addresses: kouzakov@srd.sinp.msu.ru (Konstantin A. Kouzakov), studenik@srd.sinp.msu.ru (Alexander I. Studenikin)
} 
where $m_{e}$ and $m_{\nu}$ are the electron and neutrino masses, respectively. Therefore, experimental evidence for a value which is much larger than $10^{-19}$ will signalize new physics.

The nowaday laboratory searches for neutrino magnetic moments are dealing with reactor (anti)neutrino sources (MUNU [5], TEXONO [6], and GEMMA [7]). The best upper limit for $\mu_{\nu}$ obtained so far in the reactor experiments is $\mu_{\nu} \leq 3.2 \times 10^{-11}$ [7] (see also references in the review article [1]), which still exceeds by an order of magnitude the most stringent astrophysical constraint $\mu_{\nu} \leq 3 \times 10^{-12}$ [8]. Since the differential cross section (DCS) for the magnetic scattering of a neutrino with energy $E_{\nu}$ on a free electron $(\mathrm{FE})$ at energy transfer $T$ is given by [9, 10]

$$
\frac{d \sigma_{(\mu)}}{d T}=\frac{\pi \alpha^{2} \mu_{\nu}^{2}}{m_{e}^{2}}\left(\frac{1}{T}-\frac{1}{E_{\nu}}\right)
$$

where $\alpha$ is the fine-structure constant, a way to enhance the sensitivities of the experiments is to reduce the low-energy threshold of the detectors with respect to the deposited energy $T$. Note in this connection that the DCS due to the weak interaction, $d \sigma_{(w)} / d T$, is practically constant in $T$ when $E_{\nu} \gg T[10]$.

The electrons of the detector material are bound to atoms and therefore the FE picture is applicable only when $T \gg \varepsilon_{b}$, where $\varepsilon_{b}$ is the electron binding energy. If $T \sim \varepsilon_{b}$, the electron binding effects must be taken into consideration. Recently, it was claimed that at $T \sim \varepsilon_{b}$ the DCS of the inelastic magnetic neutrino scattering on atomic electrons (AE) strongly increases, as compared to the FE case (11), owing to an atomic ionization effect [11]. This means that the sensitivity of the reactor experiments searching for $\mu_{\nu}$ can be significantly enhanced. As demonstrated below, the authors of Ref. [11] incorrectly evaluated the DCS, groundlessly employing the approximation of real photons in the whole range of the final neutrino angles. In this respect, in a more recent work [12] it was argued, by means of a quantum-mechanical sum rule, that when $E_{\nu} \gg T$ and $T$ is comparable to the characteristic atomic energies the values of DCS for the AE and FE cases are almost the same. While this promising analytical method yields a physically reasonable conclusion for the one-electron case, we show in the analysis carried out below that it requires further elaboration in order to be correctly applied to many-electron atomic systems.

In the present work, we formulate a new theoretical approach which allows us to consider the magnetic neutrino scattering on atomic electrons in a 
consistent and physically transparent fashion. This approach can be traced down to that developed for the penetration of relativistic charged projectiles in matter [13]. According to it, the DCS is given by the sum of two physically distinct components-the longitudinal and the transverse, which are associated with the corresponding components of the force imposed by the neutrino on electrons with respect to the direction of the momentum trans-

fer $\mathbf{q}$. Such a decomposition also enables us to clearly distinguish between the contributions from excited atomic states taken into account in Refs. [11] and [12], respectively, and to inspect consistently the results of those studies. The developed theory is then numerically realized in the case of magnetic neutrino-impact ionization $(\nu I I)$ of a hydrogen-like $\mathrm{Ge}^{+31}$ ion. This specific case models the $K$-shell ionization of Ge by neutrino impact, which is relevant to the reactor experiments [6, 7].

The article is organized as follows. Sec. 2 delivers general theory for the magnetic neutrino scattering on atomic electrons as well as a critical account of the theoretical studies published recently [11, 12]. Numerical calculations are presented and analyzed in Sec. 3. The conclusions are drawn in Sec. 4 . The units $\hbar=c=1$ are used throughout unless otherwise stated.

\section{Theory of magnetic neutrino-impact excitation and ionization of atoms}

We consider the process where a neutrino with energy $E_{\nu}$ and momentum $\mathbf{p}_{\nu}$ scatters on an atom at energy-momentum transfer $q=(T, \mathbf{q})$. In what follows we focus on the role that plays in this process the interaction of the neutrino magnetic moment with atomic electrons, assuming the nucleus to be infinitely massive. The latter assumption is reasonable if $T \gg E_{\nu}^{2} / M$, where $M$ is the nuclear mass, in which case the recoil of the atom as a whole can be safely neglected and the coherent neutrino-atom collisions (without changing the atomic state) are of no relevance. The atomic target is supposed to be unpolarized and in its ground state $|0\rangle$ with the corresponding energy $E_{0}$. We also suppose that $T \ll m_{e}$ and $\alpha Z \ll 1$, where $Z$ is the nuclear charge, so that both the initial and the final electronic systems can be treated nonrelativistically. The incident and final neutrino states are described by the Dirac spinors assuming $m_{\nu} \approx 0$. 


\subsection{The differential cross section}

In the low-energy limit the neutrino electromagnetic vertex associated with the neutrino magnetic moment has the form

$$
\Lambda_{(\mu)}^{i}=\frac{\mu_{\nu}}{2 m_{e}} \sigma^{i k} q_{k} .
$$

It should be noted that the $\mu_{\nu}$ related contribution to the neutrino-atom scattering does not interfere with that due to weak interaction because in contrast to the latter it couples neutrino states with different helicities. Employing first-order perturbation theory and using the photon propagator in the Coulomb gauge, the transition matrix element for the considered process according to Eq. (2) is given by

$$
\begin{aligned}
M_{f i}^{(\mu)}= & \frac{2 \pi \alpha \mu_{\nu}}{m_{e} \mathbf{q}^{2}}\left(\bar{u}_{\mathbf{p}_{\nu}-\mathbf{q} \lambda_{f}} u_{\mathbf{p}_{\nu} \lambda_{i}}\right)\left\{\left(2 E_{\nu}-T\right)\left\langle n\left|\sum_{j=1}^{Z} e^{i \mathbf{q} \cdot \mathbf{r}_{j}}\right| 0\right\rangle\right. \\
& \left.+\frac{2 \mathbf{q} \times\left[\mathbf{p}_{\nu} \times \mathbf{q}\right]}{T^{2}-\mathbf{q}^{2}} \cdot\left\langle n\left|\sum_{j=1}^{Z} \boldsymbol{\alpha}_{j} e^{i \mathbf{q} \cdot \mathbf{r}_{j}}\right| 0\right\rangle\right\}
\end{aligned}
$$

where $u_{\mathbf{p} \lambda}$ is the spinor amplitude of the neutrino state with momentum $\mathbf{p}$ and helicity $\lambda, \boldsymbol{\alpha}_{j}$ the current operator of the $j$ th electron, and $|n\rangle$ the final atomic state. Using Eq. (3), the double differential cross section can be presented as

$$
\frac{d^{2} \sigma_{(\mu)}}{d T d Q}=\left(\frac{d^{2} \sigma_{(\mu)}}{d T d Q}\right)_{\|}+\left(\frac{d^{2} \sigma_{(\mu)}}{d T d Q}\right)_{\perp},
$$

where $Q=\mathbf{q}^{2}, T^{2} \leq Q \leq\left(2 E_{\nu}-T\right)^{2}$, and

$$
\begin{aligned}
\left(\frac{d^{2} \sigma_{(\mu)}}{d T d Q}\right)_{\|}= & \frac{\pi \alpha^{2} \mu_{\nu}^{2}}{m_{e}^{2}} \frac{\left(2 E_{\nu}-T\right)^{2}}{4 E_{\nu}^{2} Q}\left(1-\frac{T^{2}}{Q}\right) \\
& \times \sum_{n}|\langle n|\rho(-\mathbf{q})| 0\rangle|^{2} \delta\left(T-E_{n}+E_{0}\right), \\
\left(\frac{d^{2} \sigma_{(\mu)}}{d T d Q}\right)_{\perp}= & \frac{\pi \alpha^{2} \mu_{\nu}^{2}}{m_{e}^{2}} \frac{\left(2 E_{\nu}-T\right)^{2}}{4 E_{\nu}^{2} Q}\left[1-\frac{Q}{\left(2 E_{\nu}-T\right)^{2}}\right] \\
& \times \sum_{n}\left|\left\langle n\left|\hat{\mathbf{e}}_{\perp} \cdot \mathbf{j}(-\mathbf{q})\right| 0\right\rangle\right|^{2} \delta\left(T-E_{n}+E_{0}\right) .
\end{aligned}
$$


In Eqs. (5) and (6),$\rho(-\mathbf{q})$ and $\mathbf{j}(-\mathbf{q})$ are the Fourier transforms of the electron density and current density operators, respectively,

$$
\begin{gathered}
\rho(-\mathbf{q})=\sum_{j=1}^{Z} e^{i \mathbf{q} \cdot \mathbf{r}_{j}}, \\
\mathbf{j}(-\mathbf{q})=-\frac{i}{2 m_{e}} \sum_{j=1}^{Z}\left(e^{i \mathbf{q} \cdot \mathbf{r}_{j}} \nabla_{j}+\nabla_{j} e^{i \mathbf{q} \cdot \mathbf{r}_{j}}\right),
\end{gathered}
$$

and the unit vector $\hat{\mathbf{e}}_{\perp}$ is directed along the $\mathbf{p}_{\nu}$ component which is perpendicular to $\mathbf{q}\left(\hat{\mathbf{e}}_{\perp} \cdot \mathbf{q}=0\right)$. The sums in Eqs. (5) and (6) run over all atomic states $|n\rangle$, with $E_{n}$ being their energies, and, since the ground state $|0\rangle$ is unpolarized, do not depend on the direction of $\mathbf{q}$.

The representation (4) is similar to that for the case of charged projectiles [13]. The longitudinal term (5) is associated with atomic excitations induced by the force that the neutrino magnetic moment imposes on electrons in the direction parallel to $\mathbf{q}$. And in the case of nonrelativistic projectiles, only such excitations are of significance. In the present case, however, the projectile is ultrarelativistic. The transverse term (6) corresponds to the exchange of a virtual photon which is polarized as a real one, that is, perpendicular to q. It can be noted that it resembles a photoabsorption process when $Q \rightarrow T^{2}$ and the virtual-photon four-momentum thus approaches a physical value, $q^{2} \rightarrow 0$. An important feature is that, due to selections rules, Eqs. (5) and (66) involve different, nonintersecting sets of atomic states (see Ref. [13] for detail). For this reason they do not interfere. Another marked difference between the terms (5) and (6) is that the first vanishes in the forward direction, when $Q=T^{2}$, while the second in the backward direction, when $Q=\left(2 E_{\nu}-T\right)^{2}$.

In the reactor experiments one typically measures the DCS which derives from (4) upon integrating over $Q$ :

$$
\frac{d \sigma_{(\mu)}}{d T}=\int_{T^{2}}^{\left(2 E_{\nu}-T\right)^{2}} \frac{d^{2} \sigma_{(\mu)}}{d T d Q} d Q=\left(\frac{d \sigma_{(\mu)}}{d T}\right)_{\|}+\left(\frac{d \sigma_{(\mu)}}{d T}\right)_{\perp} .
$$

Below we separately analyze the longitudinal and transverse components of Eq. (9) assuming $E_{\nu} \gg T$, which situation is usual for the reactor experiments. 


\subsection{The longitudinal component of the differential cross section}

When integrating Eq. (5) over $Q$, we neglect the contribution of the term $\propto T^{2} / Q$ that brings only a minor correction to the integral, so that [12]

$$
\left(\frac{d \sigma_{(\mu)}}{d T}\right)_{\|}=\frac{\pi \alpha^{2} \mu_{\nu}^{2}}{m_{e}^{2}} \int_{T^{2}}^{4 E_{\nu}^{2}} S(T, Q) \frac{d Q}{Q},
$$

where we have introduced the so-called dynamical structure factor [14]

$$
S(T, Q)=\sum_{n}|\langle n|\rho(-\mathbf{q})| 0\rangle|^{2} \delta\left(T-E_{n}+E_{0}\right) .
$$

The latter is related to the density-density or polarization Green's function $F$ as follows:

$$
\begin{aligned}
S(T, Q) & =\frac{1}{\pi} \operatorname{Im} F\left(T+E_{0}, Q\right), \\
F\left(T+E_{0}, Q\right) & =\left\langle 0\left|\rho(\mathbf{q}) \frac{1}{T+E_{0}-H-i 0} \rho(-\mathbf{q})\right| 0\right\rangle \\
& =\sum_{n} \frac{|\langle n|\rho(-\mathbf{q})| 0\rangle|^{2}}{T-E_{n}+E_{0}-i 0},
\end{aligned}
$$

where $H$ is the atomic Hamiltonian. Using definitions (11) and (12), it is straightforward to obtain the dispersion relation

$$
F(E, Q)=\frac{1}{\pi} \int_{0}^{\infty} \frac{\operatorname{Im} F\left(E^{\prime}, Q\right)}{E-E^{\prime}-i 0} d E^{\prime} .
$$

The dynamical structure factor (11) can be expressed in terms of the generalized oscillator strengths $f_{n}$ [15],

$$
\begin{aligned}
S(T, Q) & =\frac{Q}{2 m_{e} T} \sum_{n} f_{n}(Q) \delta\left(T-E_{n}+E_{0}\right), \\
f_{n}(Q) & =\frac{2 m_{e}}{Q}\left(E_{n}-E_{0}\right)|\langle n|\rho(-\mathbf{q})| 0\rangle|^{2},
\end{aligned}
$$

and thus it satisfies the Bethe sum rule [15, 16]

$$
\int_{0}^{\infty} S(T, Q) \frac{d T^{2}}{Q}=\frac{Z}{m_{e}} .
$$


However, it is not of much help for performing the integration over $Q$ in Eq. (10). For the latter purpose the following momentum-transfer dispersion relation was formulated in Ref. [12] (cf. Eq. (13)):

$$
F(E, Q)=\frac{1}{\pi} \int_{0}^{\infty} \frac{\operatorname{Im} F\left(E, Q^{\prime}\right)}{Q^{\prime}-Q-i 0} d Q^{\prime},
$$

provided the energy $E$ is above the ionization threshold. Consider the limit $Q \rightarrow 0$. The electron density operator (7) at $\mathbf{q}=0$ is by definition $\rho(0)=Z$ and hence

$$
F\left(T+E_{0}, 0\right)=\left\langle 0\left|\rho(0) \frac{1}{T+E_{0}-H-i 0} \rho(0)\right| 0\right\rangle=\frac{Z^{2}}{T} .
$$

Using it in Eq. (16) when $Q=0$, we arrive at the sum rule (cf. Eq. (15))

$$
\int_{0}^{\infty} S(T, Q) \frac{d Q}{Q}=\frac{Z^{2}}{T}
$$

Following the method of Ref. [12], which implies the use of Eq. (17) for evaluating the integral in Eq. (10) under assumptions of small $T$ and large $E_{\nu}$, we get

$$
\left(\frac{d \sigma_{(\mu)}}{d T}\right)_{\|} \simeq \frac{\pi \alpha^{2} \mu_{\nu}^{2}}{m_{e}^{2}} \frac{Z^{2}}{T}=Z^{2} \frac{d \sigma_{(\mu)}^{\mathrm{FE}}}{d T},
$$

where $d \sigma_{(\mu)}^{\mathrm{FE}} / d T$ is given by Eq. (1) in the limit $T / E_{\nu} \rightarrow 0$.

Thus, using the dispersion relation (16), one arrives at Eq. (18) where the factor of $Z^{2}$ occurs, which means that the atomic effects result in a coherent enhancement of the DCS as compared to the case of $Z$ free electrons, where a typical incoherent-scattering factor of $Z$ is encountered (the same as, for instance, in the Compton scattering). This indicates that Eq. (16) is not directly applicable to atoms with more than one electron. At the same time, under some modification the method of Ref. [12] can be viable, for instance, within the mean-field model of many-electron atomic systems, where the occupied one-electron orbitals independently contribute to the $\nu I I$ process. In such a case, one employs the sum rule (17) for each electron individually (the proving of one-electron momentum-transfer sum rules is suggested in Ref. [12]). Clearly, this procedure ensures a physically-grounded factor of $Z$ in Eq. (18) after collecting together the one-electron contributions. Note that the electron correlation effects beyond the mean-field approximation crucially influencing multiple excitation and ionization processes usually slightly affect those of single ionization. 


\subsection{The transverse component of the differential cross section}

As demonstrated below, it is the case which is relevant to the work [11]. Integrating Eq. (6) over $Q$ and dropping the insignificant term $\propto Q /\left(2 E_{\nu}-\right.$ $T)^{2}$, we get

$$
\left(\frac{d \sigma_{(\mu)}}{d T}\right)_{\perp}=\frac{\pi \alpha^{2} \mu_{\nu}^{2}}{m_{e}^{2}} \int_{T^{2}}^{4 E_{\nu}^{2}} R(T, Q) \frac{d Q}{Q},
$$

where the function $R$ is defined as

$$
R(T, Q)=\sum_{n}\left|\left\langle n\left|\hat{\mathbf{e}}_{\perp} \cdot \mathbf{j}(-\mathbf{q})\right| 0\right\rangle\right|^{2} \delta\left(T-E_{n}+E_{0}\right) .
$$

Similarly to the dynamical structure factor (11), it can be related to the current-current Green's function $L$ (cf. Eq. (12)):

$$
\begin{aligned}
R(T, Q) & =\frac{1}{\pi} \operatorname{Im} L\left(T+E_{0}, Q\right), \\
L\left(T+E_{0}, Q\right) & =\left\langle 0\left|j_{\perp}(\mathbf{q}) \frac{1}{T+E_{0}-H-i 0} j_{\perp}(-\mathbf{q})\right| 0\right\rangle \\
& =\sum_{n} \frac{\left|\left\langle n\left|j_{\perp}(-\mathbf{q})\right| 0\right\rangle\right|^{2}}{T-E_{n}+E_{0}-i 0},
\end{aligned}
$$

where $j_{\perp}( \pm \mathbf{q})=\hat{\mathbf{e}}_{\perp} \cdot \mathbf{j}( \pm \mathbf{q})$.

Since $\mathbf{j}=(\rho \mathbf{v}+\mathbf{v} \rho) / 2$, with $\mathbf{v}$ being the electron velocity operator, we can roughly estimate the ratio of the functions (20) and (11) as $\sim v_{a}^{2}$, where $v_{a} \ll$ 1 is a characteristic velocity of atomic electrons. A more accurate estimate can be obtained when $Q$ is small on the atomic scale. If $Q \ll 2 m_{e} \varepsilon_{b}$ one can treat Eqs. (11) and (20) in the dipole approximation (see also Ref. [12]), which yields

$$
\frac{R(T, Q)}{S(T, Q)}=\frac{T^{2}}{Q}
$$

Note that this ratio is much smaller than unity practically for all $Q$ values involved in Eqs. (10) and (19). Thus, taking into account the foregoing arguments, one might expect the transverse component to play a minor role in Eq. (9). The authors of Ref. [11], however, came to the contrary conclusion that this component dramatically enhances due to atomic ionization when $T \sim \varepsilon_{b}$. The enhancement mechanism proposed in Ref. [11] is based on an analogy with the photoionization process. As mentioned above, when $Q \rightarrow$ 
$T^{2}$ the virtual-photon momentum approaches the physical regime $q^{2}=0$. In this case, we have for the integrand in Eq. (19)

$$
\left.\frac{R(T, Q)}{Q}\right|_{Q \rightarrow T^{2}}=\frac{\sigma_{\gamma}(T)}{4 \pi^{2} \alpha T},
$$

where $\sigma_{\gamma}(T)$ is the photoionization cross section at the photon energy $T$ [17]. The limiting form (23) was used in Ref. [11] in the whole integration interval. Such a procedure is obviously incorrect, for the integrand rapidly falls down as $Q$ ranges from $T^{2}$ up to $4 E_{\nu}^{2}$, especially when $Q \gtrsim r_{a}^{-2}$, where $r_{a}$ is a characteristic atomic size (within the Thomas-Fermi model $r_{a}^{-1}=Z^{1 / 3} \alpha m_{e}$ [18]). This fact reflects a strong departure from the real-photon regime. For this reason we can classify the enhancement of the DCS determined in Ref. [11] as spurious.

\section{Numerical estimates of electron binding effects}

Let us inspect numerically the magnetic $\nu I I$ process of a hydrogenlike ion with a nuclear charge $Z=32$ that mimics the case of a $K$-electron in $\mathrm{Ge}$, which is a detector material in the reactor experiments [6, 7]. The functions (11) and (20) are calculated analytically using the well-known expressions for the generalized oscillator strengths (14) (see, for instance, Ref. [15]) and photoionization matrix elements [17] corresponding to transitions from a $1 s$ hydrogenlike state. Then, the $Q$ integrations in Eq. (9) are performed numerically. Fig. 11 shows the DCS (9), which is normalized to the FE value (11), above the ionization threshold as a function of $T / \varepsilon_{b}$, with the electron binding energy given by $\varepsilon_{b}=\alpha^{2} Z^{2} m_{e} / 2 \approx 14 \mathrm{keV}$. As can be seen, the AE results for $E_{\nu} \gg \varepsilon_{b}$ are close to the FE ones in magnitude. Such a behavior agrees both with the analytical estimates performed in Ref. [12] and with the numerical results of more rigorous, relativistic treatments for various atomic targets [19]. It can be qualitatively explained by noticing the following facts. First, in an attractive Coulomb potential there is an infinite set of bound states, with the discrete spectrum smoothly transforming into the continuum at the ionization threshold. Second, the average value of the $K$-electron momentum is $p_{e}=\alpha Z m_{e}$ and the average change in the electron momentum after ejection, $\Delta p_{e}$, is such that $\Delta p_{e}^{2}=2 m_{e} T$, which is analogous to the FE case. Thus, in contrast to the claim of Ref. [11], at small recoil-electron ener-

gies one might expect no enhancement of the sensitivity of the experiments searching for $\mu_{\nu}$. 


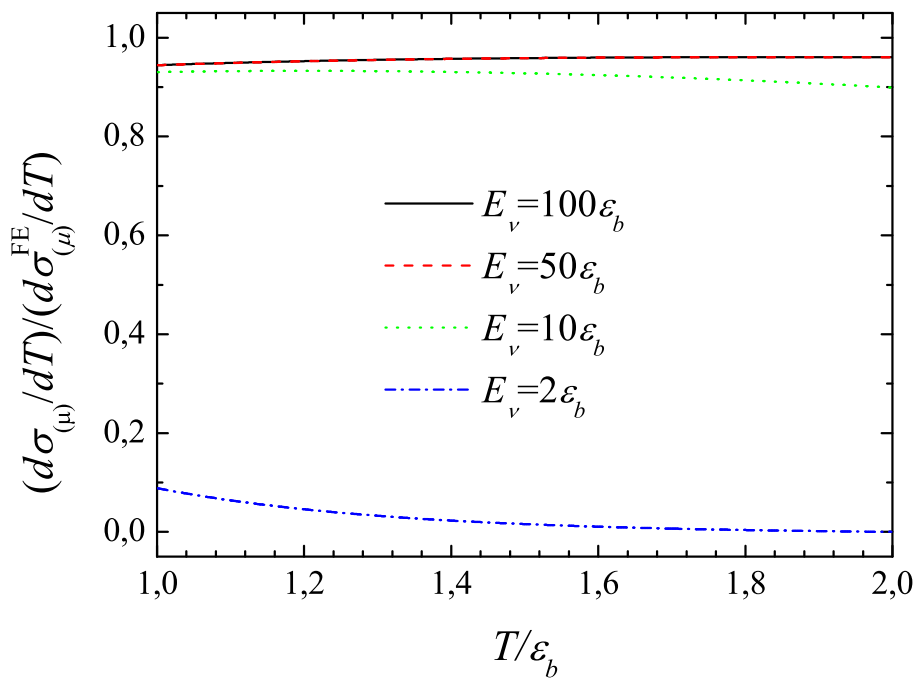

Figure 1: The DCS normalized to the FE result versus $T / \varepsilon_{b}$ for different values of $E_{\nu}$. The $E_{\nu}=50 \varepsilon_{b}$ and $E_{\nu}=100 \varepsilon_{b}$ curves are practically indistinguishable.

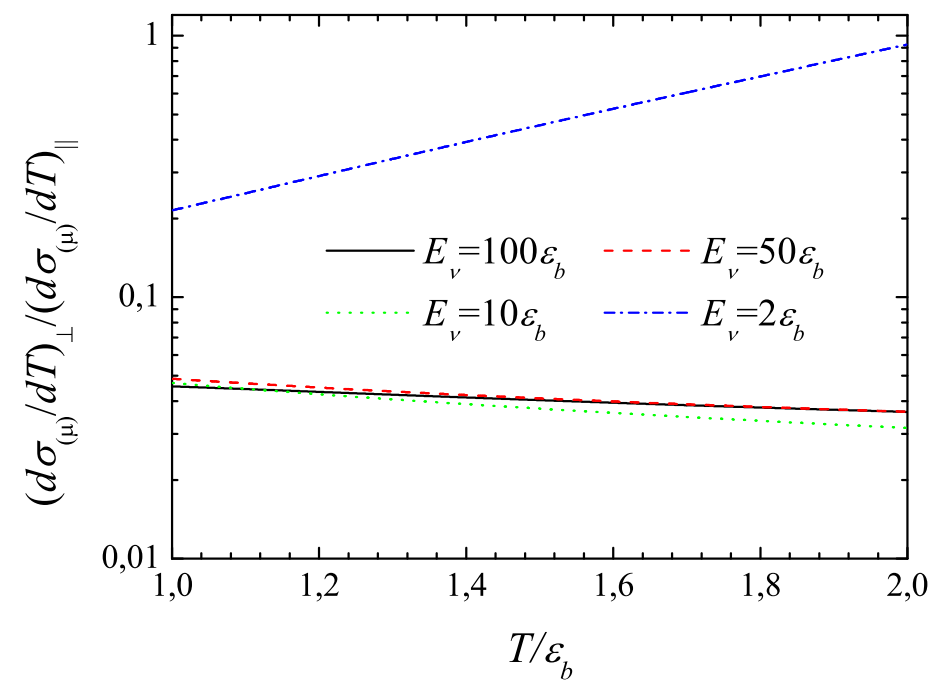

Figure 2: The dependence of the ratio between the transverse and longitudinal components of the DCS (9) on $T / \varepsilon_{b}$ at different values of $E_{\nu}$. 
Fig. 2 presents numerical results for the ratio of the transverse and longitudinal terms of Eq. (9) in the same kinematical situations as in Fig. 1. As anticipated, when $E_{\nu} \gg \varepsilon_{b}$ the relative role of the transverse component is subsidiary. Though the results for $E_{\nu}=2 \varepsilon_{b}$ are not strictly relevant to the reactor experiments, where the typical neutrino energies are much higher $(\sim 1 \mathrm{MeV})$, they are shown for illustrative purposes-in this case the contribution from the transverse excitations becomes considerable, especially as $T$ increases. This finding can be explained by Eq. (22) and different kinematical factors $1-T^{2} / Q$ and $1-Q /\left(2 E_{\nu}-T\right)^{2}$ in Eqs. (5) and (6), respectively. It also can be noted that the kinematical regime where $T$ is close to $E_{\nu}$ validates applicability of the real-photon approximation (23) in the whole kinematically available region $T^{2} \leq Q \leq\left(2 E_{\nu}-T\right)^{2}$. However, as clearly seen from Fig. 1, even in a such marginal situation the DCS behaves oppositely to the resonant-enhancement scenario of Ref. [11].

\section{Conclusions}

To summarize, we have performed a theoretical analysis of the magnetic neutrino scattering on atomic electrons. For this purpose we have divided the DCS into two components corresponding to the longitudinal and transverse atomic excitations. Incorrectness of the recent theoretical predictions assigning the atomic effects to play a significant role in the magnetic neutrino scattering [11] has been revealed. Numerical calculations of the DCS for the magnetic $\nu I I$ process of a hydrogenlike $\mathrm{Ge}^{+31}$ ion have been carried out at different neutrino energies. The results exhibited suppression of the DCS relative to the FE values, which is slight at high impact energy and pronounced when the latter is comparable to the electron binding energy.

The present results based on nonrelativistic calculations qualitatively agree with those using a relativistic Dirac-Hartree-Fock treatment of atomic electrons [19]. No enhancement of the DCS due to electron binding effects has been determined, in contrast to Ref. [11]. In this regard, the analytical approach arguing the insignificance of the atomic effects [12] might have a rich potential, provided that it is appropriately modified. Finally, it is unreasonable to expect the effects of atomic excitation and/or ionization to introduce any enhancement of the sensitivities of the experiments searching for neutrino magnetic moments. Therefore, it will be interesting to explore the role of coherent magnetic neutrino scattering on atoms in detectors, which case, 
however, requires much lower energy thresholds in the deposited energy $T$ $(\sim 100 \mathrm{eV})$ than presently attainable in the detectors $(\sim 1 \mathrm{keV})$.

\section{Acknowledgements}

We thank Victor B. Brudanin and Alexander S. Starostin for useful discussions. We are grateful to Mikhail B. Voloshin for valuable comments.

\section{References}

[1] C. Giunti and A.I. Studenikin, Phys. At. Nucl. 73 (2009) 2089, arXiv:08123646 [hep-ph].

[2] W.J. Marciano and A.I. Sanda, Phys. Lett. B 67 (1977) 303.

[3] B.W. Lee, R.E. Shrock, Phys. Rev. D 16 (1977) 1444.

[4] K. Fujikawa, R.E. Shrock, Phys. Rev. Lett. 45 (1980) 963.

[5] Z. Daraktchieva et al. (MUNU collaboration), Phys. Lett. B 615 (2005) 153, arXiv:hep-ex/0502037.

[6] H.T. Wong et al. (TEXONO collaboration), Phys. Rev. D 75 (2007) 012001.

[7] A.G. Beda et al. (GEMMA collaboration), arXiv:0906.1926 [hep-ex]; arXiv:1005.2736 [hep-ex].

[8] G.G. Raffelt, Phys. Rev. Lett. 64 (1990) 2856.

[9] G.V. Domogatskii, D.K. Nadezhin, Sov. J. Nucl. Phys. 12 (1971) 678.

[10] P. Vogel, J. Engel, Phys. Rev. D 39 (1989) 3378.

[11] H.T. Wong, H.-B. Li, S.-T. Lin, Phys. Rev. Lett. 105 (2010) 0161801, arXiv:1001.2074 [hep-ph].

[12] M.B. Voloshin, Phys. Rev. Lett. 105 (2010) 201801, arXiv:1008.2171 [hep-ph].

[13] U. Fano, Annu. Rev. Nucl. Sci. 13 (1963) 1. 
[14] L. Van Hove, Phys. Rev. 95 (1954) 249.

[15] M. Inokuti, Rev. Mod. Phys. 43 (1971) 297.

[16] H. Bethe, Ann. Physik 5 (1930) 325.

[17] A.I. Akhiezer, V.B. Berestetskii, Quantum Electrodynamics, second ed., Wiley, New York, 1965.

[18] L.D. Landau, E.M. Lifshitz, Quantum Mechanics, Non-Relativistic Theory, third ed., Pergamon, New York, 1977.

[19] V.Yu. Dobretsov, A.B. Dobrotsvetov, S.A. Fayans, Sov. J. Nucl. Phys. 55 (1992) 1180; V.I. Kopeikin, L.A. Mikaelyan, V.V. Sinev, S.A. Fayans, Phys. At. Nucl. 60 (1997) 1859; S.A. Fayans, L.A. Mikaelyan, V.V. Sinev, Phys. At. Nucl. 64 (2001) 1475. 